\title{
Added Value of Microscale Raman Chemical Analysis in Mild Traumatic Brain Injury (TBI): A Comparison with Macroscale MRI
}

Dmitry Khalenkow, ${ }^{* \dagger}$ Sam Donche, ${ }^{\dagger,+, \S}$ Kim Braeckman, ${ }^{\dagger}$ Christian Vanhove,

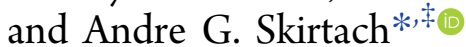

${ }^{\dagger}$ Department of Molecular Biotechnology, Faculty of Bioscience Engineering and ${ }^{\ddagger}$ INFINITY Laboratory, Faculty of Engineering and Architecture, University of Ghent, 9000 Ghent, Belgium

Supporting Information

ABSTRACT: Diffuse axonal injury and microhemorrhages, common complications after mild traumatic brain injury (mTBI), can lead to neurodegeneration and disability and have negative socioeconomic consequences. Magnetic resonance imaging (MRI) is conventionally used to study brain injuries in vivo, but microscale damage common in mTBI is challenging to detect. Raman microscopy is an effective diagnostic tool to investigate cells and tissue in a label-free manner, but the scanning mode of Raman microscopy is typically used only in vitro. Here, we show that Raman microscopy complements in vivo MRI, providing the vital information on the structural and molecular changes caused

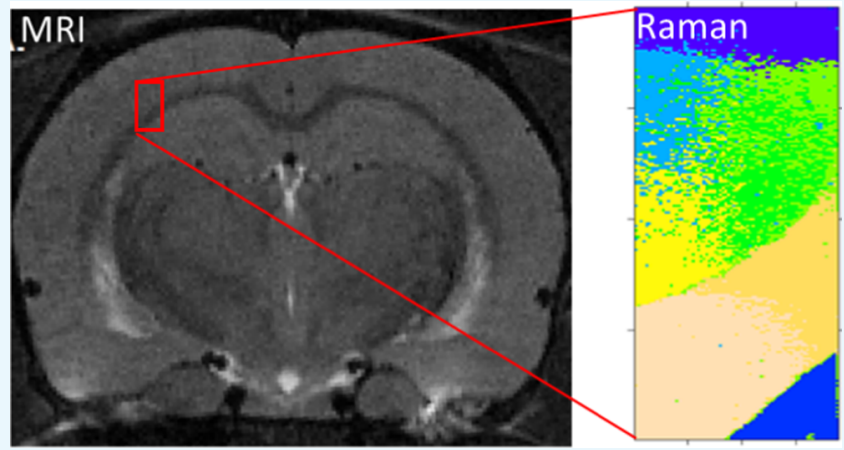
by $\mathrm{mTBI}$ in rats. We demonstrate that a method based on Raman microscopy allows us to detect structural damage invisible by conventional MRI and spot molecular changes in protein/ lipid concentrations caused by mild TBI.

\section{INTRODUCTION}

Traumatic brain injury (TBI) is the pathological alteration in brain functions caused by the impact of an object or the rapid acceleration/deceleration of the brain. In recent years, it has been recognized as an important health issue with a negative socioeconomic impact. ${ }^{1,2}$ There are several gradations of TBI, mild, moderate, and severe, based on the Glasgow Coma Scale and the severity of symptoms. ${ }^{3}$ Mild TBI (mTBI) is diagnosed in $80 \%$ of head trauma patients in, for example, the United States. ${ }^{4}$ It is a common trauma that can occur on numerous occasions: in military conflicts; as a result of sporting activities, particularly in sports such as American football or boxing; and as a result of day-to-day activities associated with possible injuries (bicycle, motorcycle, and car accidents). ${ }^{5}$ With the advancement of electrical bicycles, the danger of such occasions is only increasing. It was found that at least $30 \%$ of mTBI patients were not able to achieve full neurologic recovery and had developed prolonged cognitive and behavioral changes. ${ }^{6}$

Magnetic resonance imaging (MRI) is a technique widely used for in vivo brain imaging in clinics. ${ }^{7}$ It allows for noninvasive visualization of gray matter and white matter and detection of the damage caused by TBI, such as white matter shearing, foci of axonal injury, and small subacute hemorrhages. These brain lesions were observed with MRI in patients with moderate and severe TBI. ${ }^{9}$ However, an mTBI often shows no abnormalities on conventional MRI as it is not sensitive enough to detect the microbleedings and diffuse/ traumatic axonal injuries, which are the common complications of mTBI. ${ }^{10,11}$ We have identified another technique, Raman microscopy, which can be complementary to MRI on the microscale.

Raman microscopy can thus be a viable addition to neuroimaging tools because it allows nondestructive analysis and imaging of biological materials with spatial resolutions in the micrometer range. ${ }^{12}$ It is a label-free, optical method based on the inelastic laser light scattering from molecular vibrations inherent to any material. It is widely used in monitoring the state of materials, ${ }^{13}$ chemical analysis, ${ }^{14}$ cell biology, ${ }^{15-17}$ histological and cancer studies, ${ }^{18}$ etc. It was also shown that Raman microscopy allows label-free detection of cancer cells in the brain. ${ }^{19}$ Raman spectroscopy was also used for analysis of biochemical changes in peripheral nerves after an injury ${ }^{20}$ and to track the molecular changes on the surface of the brain hemispheres after $\mathrm{TBI}^{21}$ where temporal changes in tissue biochemistry were monitored.

In this study, a subsequent application of MRI and Raman has been carried out for the detection of mTBI. To the best of our knowledge, this has not yet been studied in detail and no analysis of deep brain structures after mTBI with Raman has been done in rats. The second and a more specific aim of this work is to investigate whether any changes in the corpus

Received: September 16, 2018

Accepted: November 20, 2018

Published: December 6, 2018 
A

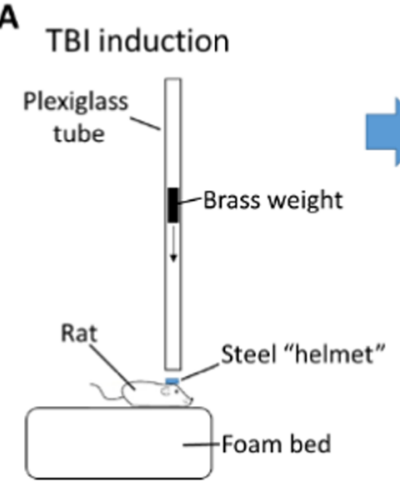

B

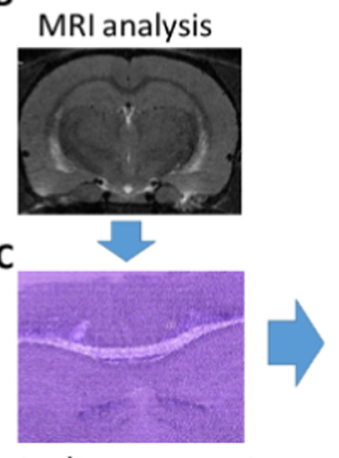

D

Raman analysis

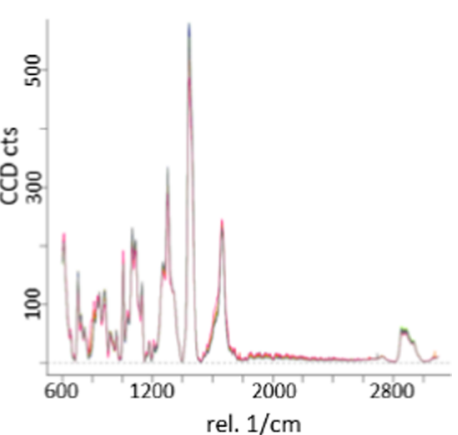

Figure 1. Schematics of experiments. (A) Marmarou's setup for inducing mTBI in rats: a real image of a rat in the device is shown in the inset. (B) MRI scans were conducted before and after TBI induction. (C) Brain slices were made with a cryotome. (D) Raman scans complimentary to the MRI test were done for detection of the damaged brain region.

callosum region can be observed after mTBI using rats as experimental models because significant changes in the volume and morphology of the corpus callosum were reported in humans after severe TBI. ${ }^{22}$

\section{RESULTS AND DISCUSSION}

Although mTBI is a major cause of disability, little is known about its underlying causes and mechanisms. Therefore, animal models are essential to study the underlying biomechanical, cellular, and molecular aspects of TBI, which cannot be studied in a clinical environment. ${ }^{23}$ In the conducted experiments, an mTBI was induced by the so-called Marmarou weight-drop model. The severity of the TBI can be controlled by adjusting the mass of the weight and the drop height. ${ }^{23}$ A replica of the Marmarou device was built for this purpose (Figure 1A). Figure $1 \mathrm{~A}-\mathrm{D}$ presents the overall schematics of experiments: first, (A) dropping the weight, then (B) MRI investigations, followed by (C) creating slices in a cryomicrotome (cryotome), and (D) scanning with a Raman microscope.

To analyze the impact of this injury, we have conducted MRI experiments (Figure 1B), which allow us to analyze the effects of $\mathrm{mTBI}$ in vivo. There are two reasons why microscopy images have an added value to MRI. First, although MRI images allow for deep in-tissue imaging, its spatial resolution is not as high as that of microscopic imaging (100 vs $1 \mu \mathrm{m}$ ). Second, MRI misses the specificity to detect the underlying biological effects of mTBI, whereas microscopic imaging might provide a valuable and complementary addition in this case. It is for these two reasons that we have chosen microscopy to provide additional means to look for details of the impact. Microscopic analysis requires the brain to be cut in thin slices (Figure 1C). Among different microscopy techniques, we have chosen Raman microscopy imaging (Figure 1D) because it allows us to image molecules, materials, and tissue in a labelfree manner by looking at the scattering of an impinged laser light vibration. We note that using Raman microscopy visualization of anatomical features in the scanned area is also possible by constructing heat maps based on the intensity of Raman peaks of interest (Figure S1).

Experiments have been conducted using both techniques on a similar area in the brain before and after TBI. The brain of the rat was scanned with conventional T2-weighted MRI before, 1 day after, and 1 week after TBI with the purpose of identifying and imaging the damaged areas. Subsequently, the brains were extracted and subjected to Raman histological analysis with the focus on the corpus callosum region.

The anatomical T2-weighted images of an animal are shown in Figure 2. Here, one can detect the baseline scan before and
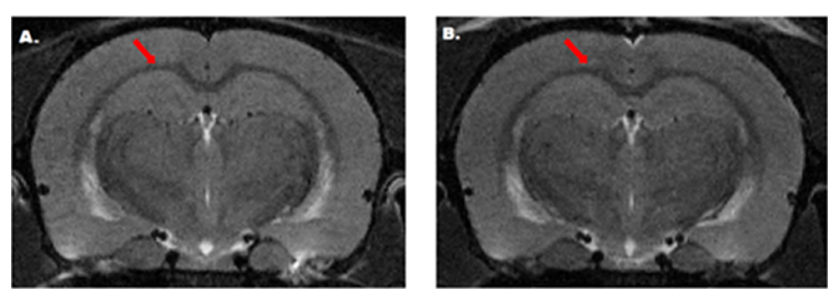

Figure 2. MRI T2-weighted brain images (A) before and (B) 1 week after the mTBI. Arrows point the corpus callosum area subjected to further investigation with Raman microscopy.

1 week after impact. Among the cut slides, we have chosen those slices where the corpus callosum and the lobes of the hippocampus are clearly visible. Because the anatomical T2 images are very similar in all animals, Figure 2 is a representative of all animals from this experiment. No significant changes were observed with conventional MRI (Figure 2) in images obtained 1 day and 1 week after the TBI compared to those of the control rats. This supports the evidence that the induced impact produces only a mild TBI.

Subsequently, Raman microscopy has been used on similar slices in an effort to search for complementarity of the in vivo MRI and a higher resolution microscopy imaging modality. In Raman microscopy/spectroscopy, a monochromatic laser light (we have used $785 \mathrm{~nm}$ laser because it generates minimum fluorescence effects, which are viewed as side effects) excites a sample, whereas the scattered light containing information about vibrational frequencies of atoms of materials is detected and analyzed. Assignment of the peaks allows us to identify molecules. It was found that the peak intensity in the 2940 $\mathrm{cm}^{-1}$ region of the Raman neuronal spectrum is proportional to the neurofilament protein concentration and that of the peak in the $2855 \mathrm{~cm}^{-1}$ region corresponds to the myelin lipid concentration. ${ }^{19}$ This allows us to track, in a label-free manner, the axon proteins/myelin turnover during peripheral nerve tissue regeneration.

The hierarchical clustering analysis has allowed us to visualize and assign the data points to different tissues present in the scanned area (Figure 3A). The subsequent hierarchical 

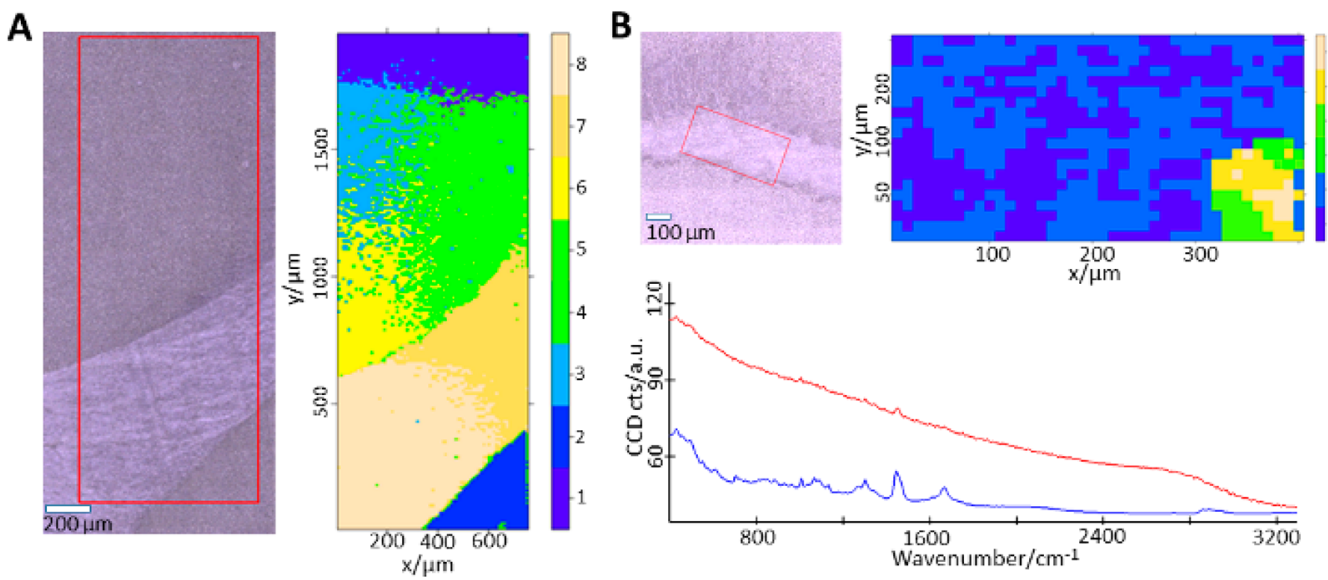

Figure 3. Light and Raman microscopy of the corpus callosum. (A) Light microscope image (left panel) and the corresponding Raman microscope image of the cortex and corpus callosum produced by hierarchical clustering analysis (right panel). The area scanned with a Raman microscope is marked with a red rectangle. (B) Light microscope image (top-left panel) and the corresponding Raman microscope image of the corpus callosum with an abnormal region (top-right panel). The mean Raman spectra of the corpus callosum (blue color) and the high intensity spectrum of the abnormal region (red color) are shown below (bottom panel).
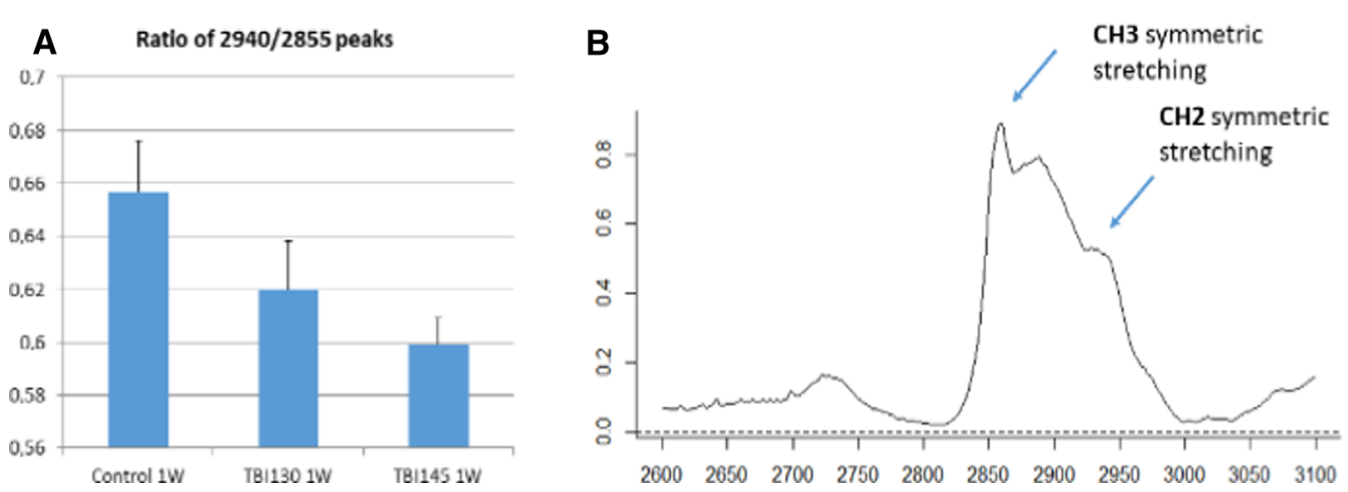

Figure 4. (A) Change in the Raman scattering intensity peak ratio at $2940 \mathrm{~cm}^{-1}$ to that at $2855 \mathrm{~cm}^{-1}$ after the diffuse TBI. The control column shows the peak ratio obtained from the corpus callosum area of a control rat (no TBI). The TBI-130 and TBI-145 columns show the peak ratios from the rats in which the mild TBI was induced by dropping the weight from 1.30 and $1.45 \mathrm{~m}$ heights, respectively. (B) Raman spectrum of the $\mathrm{CH}-$ stretching region used for the peak intensity analysis. Arrows indicate $\mathrm{CH}_{3}\left(2855 \mathrm{~cm}^{-1}\right)$ and $\mathrm{CH}_{2}\left(\right.$ at $\left.2940 \mathrm{~cm}^{-1}\right)$ peaks.

clustering analysis of data sets containing data points from the corpus callosum revealed a histologically abnormal area (15$20 \mu \mathrm{m}$ in size) in a brain slice obtained from a rat 1 week after the medium force TBI (Figure 3B).

This area had two times higher Raman signal intensity compared to that of the signal from the surrounding tissues and contained no peaks observed in the surrounding brain tissue. A similar signal pattern was noticed in the molecular fingerprint from dried rat blood (Figure S2), for which a detailed Raman analysis of blood is carried out. This suggests that the observed abnormal area may be the result of microhemorrhages common in mTBI. ${ }^{11}$ The absence of such areas in other TBI samples can be explained by either a not sufficient number of brain slices analyzed in this experiment or the fact that microhemorrhages are present only in a limited number in $\mathrm{mTBI}$.

Subsequently, the data points corresponding to the corpus callosum were compared between samples with the focus on the 2800-3000 $\mathrm{cm}^{-1}$ Raman spectral region. A comparison of spectra between the control rats and mTBI rats has revealed a significant decrease of the $2940-2855 \mathrm{~cm}^{-1}$ peak ratios after mTBI with $p=0.001299$ for TBI-130 versus the control rat. This condition was induced by a weight drop from $1.30 \mathrm{~m}$. Also, a decrease in peak ratios was observed for TBI-145 induced by a weight drop from $1.45 \mathrm{~m}$ versus the control rat with $p=0.00076$ (Figure 4). However, it is important to note that only one TBI-145 rat was investigated with Raman microscopy, as the second rat was found dead next day after the impact. Because of the ethical consideration, it was decided not to subject additional animals for the $1.45 \mathrm{~m}$ weight drop impact; therefore, it is not possible to conclude whether the observed changes in the Raman spectrum were the result of the weight drop from $1.45 \mathrm{~m}$ or can be attributed to individual differences between animals. A somewhat extended analysis is presented in Figure S3, which shows data of $2940-2855 \mathrm{~cm}^{-1}$ peak ratios for individual rats.

Whether the observed differences between control and TBI130 animals are the results of the changes because of induced TBI in all axons residing in the corpus callosum area or only in a limited number of axons will be the subject of future investigation. It can be thus concluded that Raman microscopy allows us to detect the damaged areas in brain after TBI and track the molecular changes on the tissue level. The main challenge of the presented approach is the fact that to perform such a study in the deep lying regions, like corpus callosum, cutting brain to thin slices is required, which is possible using MRI. Using Raman microscopy, this is possible with a higher resolution and sensitivity but only in vitro, which is destructive 
for animals. On the other hand, we note that once slices are cut, subsequent in vitro studies of tissue or cells using Raman spectroscopy are nondestructive and complementary to MRI because of the sensitivity and resolution. Furthermore, any detected abnormal molecular fingerprints can be extracted from a slice, cell, tissue, etc. and subsequently subjected to proteomic analysis or genomic sequencing. In addition, yet another complementarity between these techniques is linked with contrast enhancement by gold nanoparticles, which was reported for $\mathrm{MRI}^{24}$ and surface-enhanced Raman scattering $^{25,26}$ for Raman detection, in which the detection and analysis can also be done on particles. ${ }^{27,28}$

\section{CONCLUSIONS}

An approach combining MRI and Raman imaging is shown to be a viable tool to study mTBI on macro- and microscales. Raman microscopy provides the means to study mTBI at the tissue level, being complementary to MRI, which allows us to carry out analyses on the whole brain level. The TBI caused by the impact of a $450 \mathrm{~g}$ weight drop from 1.30 and $1.45 \mathrm{~m}$ heights did not produce lesions detectible with the conventional MRI, measured 1 day and 1 week after the impact. This provides a strong evidence that the applied force did not cause a severe TBI in rats. Using Raman microscopy, however, it was possible to detect even mTBI. This is evidenced by analyzing the decrease in the ratio of axon proteins to myeloid lipids as detected in the corpus callosum region after mTBI. The hierarchical clustering analysis allowed us to discriminate between different anatomical regions of the brain, detecting structural and molecular abnormalities inside the corpus callosum region, which is presumably the result of a microbleeding caused by mTBI.

\section{EXPERIMENTAL METHODS}

4.1. Materials and Methods. All studies were approved by the Animal Ethics Committee at Ghent University (ECD 17/96), and experiments were conducted in accordance with the guidelines of the European Commission (Directive 2010/ $63 / \mathrm{EU})$. The animals were group-housed and kept under controlled laboratory conditions ( $12 \mathrm{~h}$ light/dark cycle, $20-23$ ${ }^{\circ} \mathrm{C}$, and 40-60\% humidity).

4.1.1. Induction of Mild Traumatic Brain Injury. Adult female Wistar rats $(n=8,265 \pm 16 \mathrm{~g})$ were purchased from Janvier Labs (Le Genest-Saint-Isle, France). Five rats received a mild traumatic brain injury with varying severity (one from $1.0 \mathrm{~m}$ (TBI-100), two from $1.30 \mathrm{~m}$ (TBI-130), and two from $1.45 \mathrm{~m}$ (TBI-145)), and three rats were used as the control group (sham) and did not receive any impact.

Mild traumatic brain injury was induced using the Marmarou weight-drop model. ${ }^{29}$ Therefore, rats were anesthetized with a mixture of isoflurane and $\mathrm{O}_{2}$ (5\% induction and $2 \%$ maintenance) and injected with $0.05 \mathrm{mg} / \mathrm{kg}$ buprenorphine (Temgesic, Indivior) subcutaneously. After $30 \mathrm{~min}$, the heads of the rats were shaved, $100 \mu \mathrm{L}$ of $2 \%$ xylocaine (AstraZeneca) was locally injected in the scalp, and an incision was made to expose the skull. A metallic disc with a diameter of $10 \mathrm{~mm}$ and thickness of $3 \mathrm{~mm}$, which serves as a helmet, was glued onto the skull $1 / 3$ before and $2 / 3$ behind bregma. Next, the rat was placed on the custom-made foam bed with a mattress of a certain spring constant (Type E, Foam to Size, Ashland, VA) and positioned directly under a Plexiglas tube with a $450 \mathrm{~g}$ brass weight. The weight diameter and length were 18 and 210 $\mathrm{mm}$, respectively. The rat was briefly detached from anesthesia, and the weight was dropped from heights of $1,1.30$, and 1.45 $\mathrm{m}$. Immediately after the impact, the rat with the bed was moved away from the tube to prevent a second impact and the rat was reattached to the anesthesia. To reduce the hemodynamic shock, $1 \mathrm{~mL}$ of physiological solution $(0.9 \%$ $\mathrm{NaCl}$ ) was injected through a catheter that is placed in the lateral tail vein.

Subsequently, the helmet was removed and the incision was stitched. Additionally, the rats were imaged with a CT scan (XCube, Molecubes, Ghent, Belgium) to rule out any skull fractures because this is a criterion for euthanasia. To minimize the dose, a general-purpose, low-dose, single-bed position scan was performed. One day post impact, the rats received an extra dose of $0.05 \mathrm{mg} / \mathrm{kg}$ buprenorphine.

4.1.2. In Vivo Longitudinal MRI. MRI data were acquired on a $7 \mathrm{~T}$ MRI scanner (BioSpin Pharmascan 70/16, Bruker, Ettlingen, Germany) using a volume of rat brain/mouse wholebody RF coil. All rats were imaged at baseline, and all TBI rats were also imaged 1 day post injury. TBI-100, TBI-130, and the sham rats were also imaged 1 week post injury. During imaging, the animals were under $2 \%$ isoflurane anesthesia ( $5 \%$ for induction), whereas their body temperature was kept constant with a circulating warm-water-heated blanket and bubble wrap; the respiration rate was monitored with a pressure pad.

At each time point, a whole brain anatomical T2-weighted scan was acquired using a rapid acquisition with refocused echoes (RARE) sequence: $\mathrm{TR}=5.5 \mathrm{~s}$, $\mathrm{TE}=37 \mathrm{~ms}$, RARE factor $=8, \mathrm{FOV}=2.5 \times 2.5 \mathrm{~cm}^{2}$, and in-plane resolution of $109 \times 109 \mu \mathrm{m}^{2}$ with $600 \mu \mathrm{m}$ thick slices. There were 45 slices scanned, whereas the acquisition time was $12 \mathrm{~min}$.

4.1.3. Raman Histological Analysis. 4.1.3.1. Sample Preparation. One week later, rats were euthanized with 100 $\mathrm{mg} / \mathrm{kg}$ sodium pentobarbital (20\%) (Kela NV, Hoogstraten, Belgium) and brains were extracted from their skulls. This was immediately followed by snap freezing of the brains in 2methylbutaan (Reagent-Plus 99\%, Sigma Aldrich), which was cooled with liquid nitrogen. Sections were made with slice thickness $20 \mu \mathrm{m}$ with a cryomicrotome (CM3050 S, Leica Microsystems, Belgium). Tissue slices were put on quartz slides (Ted Pella, Inc.) and fixed for 10 min with $4 \%$ formaldehyde (4078-9001, Klinipath). Tissue slices were submerged in the Hank's balanced salt solution (14025050, ThermoFisher) to avoid tissue detachment from slides and scanned with a Raman microscope. Raman microscopy analysis was conducted on corpus callosum areas.

4.1.3.2. Data Acquisition with Raman Microscopy. The Raman microscope (Alpha300R+) was equipped with a 785 nm laser (Toptica, Munich, Germany) and a CCD camera (ANDOR iDus 401 BR-DD, Belfast, Great Britain) cooled to $-72{ }^{\circ} \mathrm{C}$. The laser power was set to $225 \mathrm{~mW}$, whereas a water immersion $20 \times / 0.6$ NA objective (Nikon) was used for scanning. Tissue was mapped with a lateral resolution of 10 $\mu \mathrm{m} /$ pixel and an integration time of $1.5 \mathrm{~s}$ per data point. A total of 2500 spectra per slice were recorded.

4.1.3.3. Data Processing. The resulting Raman spectral data sets were processed in $\mathrm{R}$ using the HyperSpec package. ${ }^{30}$ Background subtraction and baseline correction were done by applying the asymmetric least squares function and normalization by applying the area-under-curve method using the Baseline $^{31}$ and MALDIquant ${ }^{32}$ packages, respectively. Hierarchical clustering analysis with the Bray-Curtis dissimilarity 
matrix was used to select the data points corresponding to the corpus callosum region and detect the tissue structural and molecular abnormalities. For the peak ratio analysis, the average mean spectrum calculated per slice was used.

\section{ASSOCIATED CONTENT}

\section{S Supporting Information}

The Supporting Information is available free of charge on the ACS Publications website at DOI: 10.1021/acsomega.8b02404.

Rat blood analysis (Figure S1); raw heat maps generated by Raman area scan (Figure S2); change in the Raman scattering intensity peak ratio (Figure S3) (PDF)

\section{AUTHOR INFORMATION}

\section{Corresponding Authors}

*E-mail: Dmitry.Khalenkow@UGent.be (D.K.).

*E-mail: Andre.Skirtach@UGent.be (A.G.S.).

\section{ORCID}

Andre G. Skirtach: 0000-0002-4468-7620

\section{Present Address}

${ }^{\S}$ Faculty of Medicine, University of Ghent, 9000 Ghent, Belgium (S.D.).

\section{Author Contributions}

D.K. and S.D. performed Raman microscopy experiments, K.B. and S.D. performed MRI experiments, and C.V. and A.G.S. designed the experiments. All authors contributed to writing of this manuscript.

\section{Notes}

The authors declare no competing financial interest.

\section{ACKNOWLEDGMENTS}

This research is supported by the Special Research Fund (BOF) of Ghent University (24J201400010).

\section{REFERENCES}

(1) Thurman, D. J. The epidemiology and economics of head trauma. In Head Trauma: Basic, Preclinical, and Clinical Directions; Hayes, L. M. R., Ed.; Wiley: N.Y., 2001; pp 324-347.

(2) Leibson, C. L.; Brown, A. W.; Long, K. H.; Ransom, J. E.; Mandrekar, J.; Osler, T. M.; Malec, J. F. Medical Care Costs Associated with Traumatic Brain Injury over the Full Spectrum of Disease: A Controlled Population-Based Study. J. Neurotrauma 2012, 29, 2038-2049.

(3) Teasdale, G.; Jennett, B. Assessment of coma and impaired consciousness - practical scale. Lancet 1974, 304, 81-84.

(4) CDC, Injury, prevention, \& control: traumatic brain injury. Center for Disease Control and Prevention, 2010. http://www.cdc. gov/traumaticbraininjury/statistics.html.

(5) Thurman, D. J.; Branche, C. M.; Sniezek, J. E. The epidemiology of sports-related brain injuries in the United States: recent developments. J. Head Trauma Rehabil. 1998, 13, 1-8.

(6) Daneshvar, D. H.; Riley, D. O.; Nowinski, C. J.; McKee, A. C.; Stern, R. A.; Cantu, R. C. Long-Term Consequences: Effects on Normal Development Profile After Concussion. Phys. Med. Rehabil. Clin. N. Am. 2011, 22, 683-700.

(7) Tax, C. M. W.; Westin, C. F.; Dela Haije, T.; Fuster, A.; Viergever, M. A.; Calabrese, E.; Florack, L.; Leemans, A. Quantifying the brain's sheet structure with normalized convolution. Med. Image Anal. 2017, 39, 162-177.

(8) Mittl, R. L.; Grossman, R. I.; Hiehle, J. F.; Hurst, R. W.; Kauder, D. R.; Gennarelli, T. A.; Alburger, G. W. Prevalence of MR evidence of diffuse axonal injury in patients with mild head-injury and normal head CT findings. Am. J. Neuroradiol. 1994, 15, 1583-1589.

(9) Shenton, M. E.; Hamoda, H. M.; Schneiderman, J. S.; Bouix, S.; Pasternak, O.; Rathi, Y.; Vu, M. A.; Purohit, M. P.; Helmer, K.; Koerte, I.; Lin, A. P.; Westin, C. F.; Kikinis, R.; Kubicki, M.; Stern, R. A.; Zafonte, R. A review of magnetic resonance imaging and diffusion tensor imaging findings in mild traumatic brain injury. Brain Imaging Behav. 2012, 6, 137-192.

(10) Matsushita, M.; Hosoda, K.; Naitoh, Y.; Yamashita, H.; Kohmura, E. Utility of diffusion tensor imaging in the acute stage of mild to moderate traumatic brain injury for detecting white matter lesions and predicting long-term cognitive function in adults Clinical article. J. Neurosurg. 2011, 115, 130-139.

(11) Park, J. H.; Park, S. W.; Kang, S. H.; Nam, T. K.; Min, B. K.; Hwang, S. N. Detection of Traumatic Cerebral Microbleeds by Susceptibility-Weighted Image of MRI. J. Korean Neurosurg. Soc. 2009, 46, 365-369.

(12) Krafft, C.; Popp, J. The many facets of Raman spectroscopy for biomedical analysis. Anal. Bioanal. Chem. 2015, 407, 699-717.

(13) Bratashov, D. N.; Masic, A.; Yashchenok, A. M.; Bedard, M. F.; Inozemtseva, O. A.; Gorin, D. A.; Basova, T.; Sievers, T. K.; Sukhorukov, G. B.; Winterhalter, M.; Mohwald, H.; Skirtach, A. G. Raman imaging and photodegradation study of phthalocyanine containing microcapsules and coated particles. J. Raman Spectrosc. 2011, 42, 1901-1907.

(14) Kneipp, K.; Kneipp, H.; Itzkan, I.; Dasari, R. R.; Feld, M. S. Ultrasensitive chemical analysis by Raman spectroscopy. Chem. Rev. 1999, 99, 2957-2976.

(15) Matthäus, C.; Chernenko, T.; Newmark, J. A.; Warner, C. M.; Diem, M. Label-free detection of mitochondrial distribution in cells by nonresonant Raman microspectroscopy. Biophys. J. 2007, 93, 668673.

(16) Yashchenok, A.; Masic, A.; Gorin, D.; Shim, B. S.; Kotov, N. A.; Fratzl, P.; Mohwald, H.; Skirtach, A. Nanoengineered Colloidal Probes for Raman-based Detection of Biomolecules inside Living Cells. Small 2013, 9, 351-356.

(17) Baker, M. J.; Goodacre, R.; Sammon, C.; Marques, M. P.; Gardner, P.; Tipping, W.; Sule-Suso, J.; Wood, B.; Byrne, H. J.; Hermes, M.; Matousek, P.; Campbell, C. J.; El-Mashtoly, S.; Frost, J.; Phillips, C.; Diem, M.; Kohler, A.; Lau, K.; Kazarian, S.; Petrich, W.; Lloyd, G.; Delfino, I.; Cinque, G.; Isabelle, M.; Stone, N.; Kendall, C.; Jamieson, L.; Perez-Guaita, D.; Clark, L.; Gerwert, K.; Notingher, I.; Quaroni, L.; Bhargava, R.; Meade, A.; Lyng, F. Single cell analysis/ data handling: general discussion. Faraday Discuss. 2016, 187, 299327.

(18) Diem, M.; Mazur, A.; Lenau, K.; Schubert, J.; Bird, B.; Miljkovic, M.; Krafft, C.; Popp, J. Molecular pathology via IR and Raman spectral imaging. J. Biophotonics 2013, 6, 855-886.

(19) Jermyn, M.; Mok, K.; Mercier, J.; Desroches, J.; Pichette, J.; Saint-Arnaud, K.; Bernstein, L.; Guiot, M. C.; Petrecca, K.; Leblond, F. Intraoperative brain cancer detection with Raman spectroscopy in humans. Sci. Transl. Med. 2015, 7, 274 ra 19.

(20) Morisaki, S.; Ota, C.; Matsuda, K.; Kaku, N.; Fujiwara, H.; Oda, R.; Ishibashi, H.; Kubo, T.; Kawata, M. Application of Raman spectroscopy for visualizing biochemical changes during peripheral nerve injury in vitro and in vivo. J. Biomed. Opt. 2013, 18, No. 116011.

(21) Surmacki, J. M.; Ansel-Bollepalli, L.; Pischiutta, F.; Zanier, E. R.; Ercole, A.; Bohndiek, S. E. Label-free monitoring of tissue biochemistry following traumatic brain injury using Raman spectroscopy. Analyst 2017, 142, 132-139.

(22) Tomaiuolo, F.; Carlesimo, G. A.; Di Paola, M.; Petrides, M.; Fera, F.; Bonanni, R.; Formisano, R.; Pasqualetti, P.; Caltagirone, C. Gross morphology and morphometric sequelae in the hippocampus, fornix, and corpus callosum of patients with severe non-missile traumatic brain injury without macroscopically detectable lesions: a T1 weighted MRI study. J. Neurol. Neurosurg. Psychiatry 2004, 75, 1314-1322.

(23) Xiong, Y.; Mahmood, A.; Chopp, M. Animal models of traumatic brain injury. Nat. Rev. Neurosci. 2013, 14, 128-142. 
(24) Vistain, L. F.; Rotz, M. W.; Rathore, R.; Preslar, A. T.; Meade, T. J. Targeted delivery of gold nanoparticle contrast agents for reporting gene detection by magnetic resonance imaging. Chem. Commun. 2016, 52, 160-163.

(25) Kneipp, J.; Kneipp, H.; Kneipp, K. SERS - a single-molecule and nanoscale tool for bioanalytics. Chem. Soc. Rev. 2008, 37, 10521060.

(26) Ko, H.; Tsukruk, V. V. Nanoparticle-decorated nanocanals for surface-enhanced Raman scattering. Small 2008, 4, 1980-1984.

(27) (a) Fateixa, S.; Nogueira, H. I. S.; Trindade, T. Surfaceenhanced Raman scattering spectral imaging for the attomolar range detection of crystal violet in contaminated water. ACS Omega 2018, 3, 4331-4341. (b) Abalde-Cela, S.; Aldeanueva-Potel, P.; Mateo-Mateo, C.; Rodriguez-Lorenzo, L.; Alvarez-Puebla, R. A.; Liz-Marzan, L. Surface-enhanced Raman scattering biomedical applications of plasmonic colloidal particles. J. R. Soc., Interface 2010, 7, S435-S450.

(28) Yashchenok, A. M.; Borisova, D.; Parakhonskiy, B. V.; Masic, A.; Pinchasik, B. E.; Mohwald, H.; Skirtach, A. G. Nanoplasmonic smooth silica versus porous calcium carbonate bead biosensors for detection of biomarkers. Ann. Phys. 2012, 524, 723-732.

(29) Marmarou, A.; Foda, M. A. A.; Vandenbrink, W.; Campbell, J.; Kita, H.; Demetriadou, K. A new model of diffuse brain injury in Rats. 1. Pathophysiology and biomechanics. J. Neurosurg. 1994, 80, 291300.

(30) Beleites, C. S. V. hyperSpec: A Package to Handle Hyperspectral Data Sets in R, R package version 0.98-20130516. http://hyperspec.RForge.R-project.org, 2013.

(31) Eilers, P. H. C.; Boelens, H. F. M.. Baseline Correction with Asymmetric Least Squares Smoothinghttps://zanran_storage.s3. amazonaws.com/www.science.uva.nl/ContentPages/443199618.pdf, 2005.

(32) Gibb, S.; Strimmer, K. MALDIquant: a versatile R package for the analysis of mass spectrometry data. Bioinformatics 2012, 28, 2270-2271. 